\title{
Screening and quantification of the encapsulation of dyes in supramolecular particles
}

\author{
Luis A. Prieto-Costas, Logan Milton, Carla M. Quiñones \& José M. Rivera*
}

Department of Chemistry and Molecular Sciences Research Center, University of

Puerto Rico at Río Piedras, San Juan, PR, 00926. E-mail: jose.rivera151@upr.edu

\section{TOC graphic}
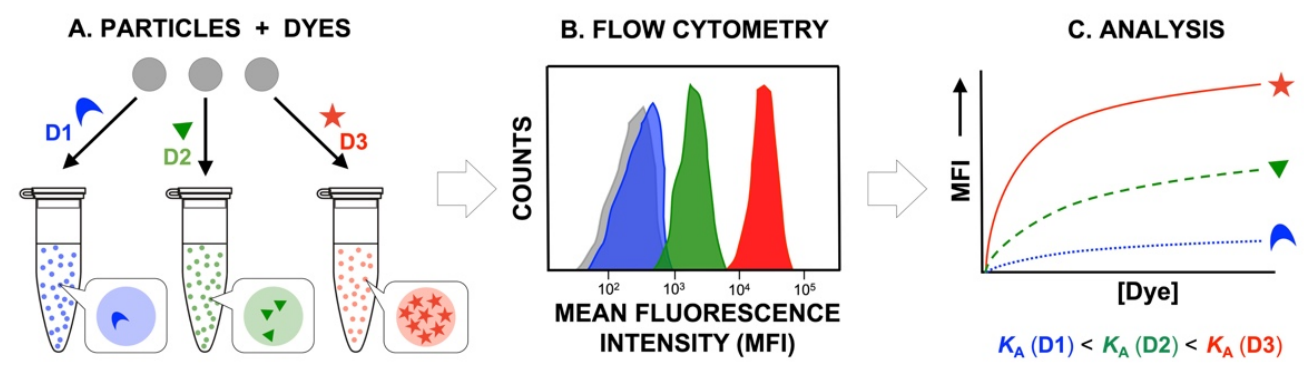

\section{Abstract}

The encapsulation of therapeutic agents, like drugs and vaccines, into colloidal particles, offers an attractive strategy to enhance their efficacy. Previously, we reported the development of guanosine-based supramolecular colloidal particles suitable for encapsulating a broad array of guests ranging from small molecule drugs, like doxorubicin, to proteins, like GFP. Many biomedical applications of such particles require a precise determination of the amount of encapsulated therapeutic agents. Despite the many publications describing the development of particle-based delivery systems, a general method for the precise and quick quantification of the encapsulated payload is still lacking. Here we report a method based on flow cytometry (FC) measurements complexes made from guanosine-based particles and a variety of commercially available fluorescent dyes. This method allows us to determine the apparent affinities of such dyes for two variants of these particles, which in turn unveils insightful structure affinity relationships. In contrast to current methods, such as those that rely on fluorescence microscopy based on measurements of absorption/fluorescence of dissolved particles or on the supernatant of the solution, the reported method is suitable for high throughput screening and more reproducible results. The protocol described here should be applicable to a wide variety of colloidal particles being developed around the world. Our group is currently expanding the scope to quantify the encapsulation of other molecules of biomedical interest, such as proteins and nucleic acids. 


\section{Introduction}

The encapsulation of therapeutic agents, like drugs and vaccines, into colloidal particles offers an attractive strategy to enhance their efficacy. Examples include micelles, liposomes, ${ }^{1,2}$ polymersomes, ${ }^{3}$ and inorganic-based particles. There is an increasing need to quantitate the affinities of complexed molecules in colloidal suspensions of macromolecules and nano/microparticles due to their increasing importance in drug delivery. ${ }^{4}$ More recent findings of the prevalence and importance of biological condensates also provide impetus to the development of versatile methods for the quantification of the partition of small molecules and macromolecules into colloidal particles. $^{5}$

Despite the many publications describing the development of particle-based delivery systems, a general method for the precise and quick quantification of encapsulated payload is still lacking. The main objectives in this study are: (1) to develop an efficient method to quantify the complexation of small molecules to colloidal microparticles; and (2) to apply the method to inquire about structural-property relationships between selected commercially available fluorescent dyes and Supramolecular Hacky Sacks (SHS particles). The first objective stems from the fact that current methods have a low accuracy and/or low throughput nature. The second will set the basis for elaborating the SHS particles' for future applications in biomedicine, like affinity-based drug delivery and developing tools for cell-biology studies.

We have reported the use of guanosine-based particles (e.g., SHS particles) as potential carriers for drug and vaccine-delivery applications. For example, we reported the use of these particles to encapsulate the drug doxorubicin and various other molecules of biological interest, like proteins and DNA. ${ }^{6,7}$ More recently, we demonstrated the use of SHS particles to improve efficacy of DNA vaccines. ${ }^{8}$

The move towards the application of the SHS particles prompted the need for precisely determining their affinity towards molecules of interest. However, most methods to quantify loading, or encapsulation/complexation efficiency, rely on elaborate and time consuming indirect or direct methods. Many of the indirect methods involve the dissolution of particles, often using organic solvents, followed by additional manipulations extractions, centrifugations, ${ }^{9}$ and filtrations. These manipulations are then followed by quantification using a variety of methods including UV/Vis spectroscopy, ${ }^{10}$ fluorescence spectrophotometry, ${ }^{11}$ and HPLC analysis, ${ }^{12,13}$ among others. ${ }^{14}$ Although the aforementioned methods provide flexibility, they suffer from slow throughput and relatively low accuracy.

Direct methods, in contrast, avoid some of the pitfalls of indirect methods, specifically, those related to the introduction of measurement errors due to multiple physical manipulations. Thus, direct spectrophotometric or fluorometric methods offer advantages over indirect methods, however, colloidal particles at the higher end of the nanoscale and beyond are not amenable for standard procedures due to scattering 
interferences and sedimentation effects. Most direct methods rely on fluorescence microscopy techniques, like CLSM, and the results are usually quantified using a parameter termed enrichment index (EI). ${ }^{5,15}$ While the use of microscopy methods avoids the effort involved in most indirect methods, they still suffer from their inherent low throughput and the potential artifacts introduced while assessing a relatively low number of particles. It is evident that there is a need to expand the repertoire of methods for direct quantification of particle loading and encapsulation efficiency.

Our group developed colloidal particles that serve as drug delivery agents. Guanosinebased particles, herein also referred to as SHS particles, are constructed from the hierarchical assembly of supramolecular G-quadruplexes (SGQs) after a change in their physical and/or chemical environment (e.g., increase in temperature, change in $\mathrm{pH}$ ). ${ }^{16,17}$ These SGQs are in turn, formed by the self-assembly of 2'-deoxyguanosine derivatives (G-monomers) in the presence of a templating salt (e.g., $\mathrm{KI}$ ). We have evaluated the encapsulation of molecules by different qualitative methods like fluorescence microscopy, but have yet to study the intrinsic affinities of these guests towards SHS particles. ${ }^{6,7}$ To achieve this, we have selected to use the FC technique, which is able to detect and measure the fluorescence of objects in the micrometer size scale.

FC is a high-throughput technique that can detect individual cells and microparticles. In the study of colloidal particles, like multilamellar vesicles, ${ }^{18}$ this technique is primarily used to determine morphology and size. The technique can be used to study the affinity constant of molecules towards cell receptors, ${ }^{19,20}$ the internalization of microparticles in cells ${ }^{21}$ and the diffusion of small molecules in multilayer particles. ${ }^{22}$ There are, however, relatively few studies of FC used for the measurement of affinity constants of particles. ${ }^{23,24}$ Temmerman and colleagues studied the affinity of fluorescent proteins with membrane lipids $^{23}$ while Liu and coworkers ${ }^{24}$ measured biomacromolecule binding in modified polystyrene microbeads; however, to the best of our knowledge, there are no studies of the use of FC to measure the affinity constants of colloidal particles towards fluorescent dyes. 


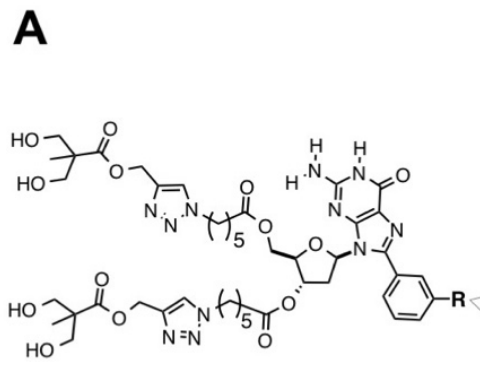

B

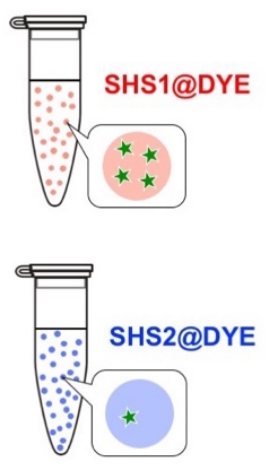

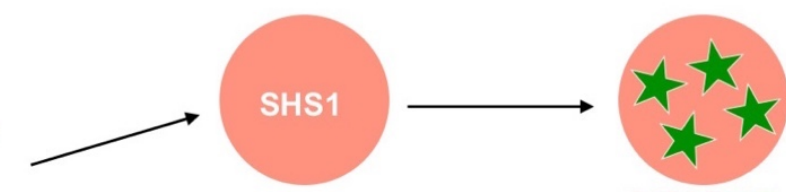

1. SELF-ASSMBLY

2. $T>40^{\circ} \mathrm{C}(L C S T)$

3. "FIXING"
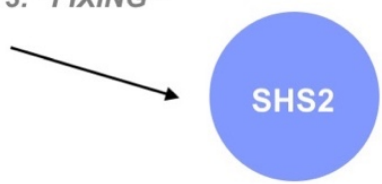

4. INCUBATION WITH DYE

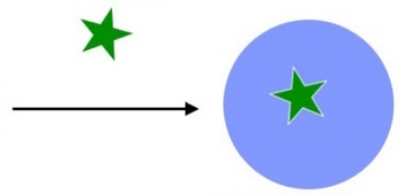

SHS2@DYE

Figure 1. Experimental overview of the encapsulation methodology of dyes in SHS particles. (A) Guanosine monomers self-assembled in the presence of a cation to form supramolecular Gquadruplexes. The self-assembled structures are further assembled by an increase in temperature to form supramolecular Hacky Sacks (SHS) or SHS particles. The temperature of formation of SHS particles in this study will be $40^{\circ} \mathrm{C}$. All experiments in this study were performed using fixed particles, meaning that they were kinetically stabilized (i.e., fixed) by lowering the ionic strength of the solution to prevent their dissolution. Dye incubation with SHS particles was done for $1 \mathrm{~h}$ at $40^{\circ} \mathrm{C}$. (B) After incubation, the SHS@Dye complexes were analyzed using flow cytometry, which enabled the construction of the saturation graphs used to calculate apparent association constants.

Here we report a method based on FC measurements of samples made from complexes of SHS particles with a variety of fluorescent dyes. The method allows us to determine the apparent affinities of such dyes for two variants of the particles, which in turn unveils insightful structural affinity relationships (Figure 1B). These findings suggest relatively weak, but specific, interactions are at play between the dyes and the constituents of the SHS particles. Our method provides quick and reproducible data in a non-destructive fashion, which represents an advantage to other direct methods, like those based on measurements of the absorption/fluorescence of dissolved particles or of the supernatant of the solution. 


\section{Results and Discussion}

Guanosine-based colloidal particles can be analyzed by FC due to their size $(\sim 1 \mu \mathrm{m})$. The monomer unit used in the formation of the particles affects the emerging properties at the microscale. Previously, we discovered that precise modifications in the G-derivatives control the temperature of formation of the colloidal particles. ${ }^{17}$ Also, the incorporation of a pH-sensitive moiety, like an imidazole, to the monomer controls the formation of SHS particles via changes in $\mathrm{pH}^{25}$ Since changes in the constituent G-derivatives leads to different macroscopic properties, we selected two G-derivatives to test the affinity of targeted molecules towards the resulting SHS particles.

The SHS particles in this study were prepared from either one of the G-derivatives 1 and 2, which differ in the group attached to the meta-carbonyl phenyl ring joined to the C8 of the guanine moiety. Derivative 1 has a conjugated imidazole group, while 2 contains an ethyl ester (Figure 1). Both compounds self-assemble to SGQs and SHS particles in PBS containing $2 \mathrm{M} \mathrm{KI}$. Subsequent reduction of the ionic strength kinetically stabilizes (or "fixes") the SHS particles, which are then suitable for the encapsulation of a wide variety of guests like dyes and proteins. For this study, kinetically stabilized SHS particles made from 1 and 2 will be termed SHS1 and SHS2, respectively.

At the microscale, the differences between these particles are the size (i.e., hydrodynamic diameter) and Z-potential (i.e., surface charge of the particle $\left.{ }^{26}\right)$. The size of the particles is $2 \mu \mathrm{m}$ and $1.3 \mu \mathrm{m}$ for SHS1 and SHS2 at $25^{\circ} \mathrm{C}$, respectively (Figure $\mathrm{S} 1$; Table S1). The Z-potential is $-16 \mathrm{mV}$ and $-11 \mathrm{mV}$ for SHS1 and SHS2 at $25^{\circ} \mathrm{C}$, respectively (Figure S1; Table S1). For both SHS1 and SHS2 parameters like monomer and salt concentration, $\mathrm{pH}$, temperature, and incubation time were kept identical to minimize undesired variability that may complicate data analysis.

We selected 14 commercially available dyes (Figure 2) previously reported to interact with DNA-quadruplexes (G4 DNA), such as porphyrins (e.g., TmPyP4), ${ }^{27}$ or guanosinebased colloidal particles, such as xanthene dyes (e.g., rhodamine B). ${ }^{7}$ Earlier we established that the SHS particles are made from SGQs, thus we hypothesized that many interactions with the selected guests would be similar to nucleic acid-based Gquadruplexes (e.g., G4-DNA/RNA). Both G4-DNA/RNA and SGQs have planar aromatic regions on their surfaces that serve as potential binding sites for aromatic molecules through end-stacking interactions (e.g., $\Pi-\pi$ interactions). Selected cationic and anionic porphyrins (TM, PIX and MPIX) have analogous planar binding surfaces that complement the G4 structure. ${ }^{28,29}$ Other smaller aromatic dyes bind to these structures via endstacking, but we must also consider the ionic charge of these dyes (positive, negative or zwitterionic). One of the binding modes of G4-DNA are the electrostatic interactions of the negative phosphate backbone with positive molecules, like the tetracationic TmPyP4. 
Although the SGQs have no phosphate backbone, the negative zeta potential of the resulting SHS particles may lead to a preference for cationic molecules.

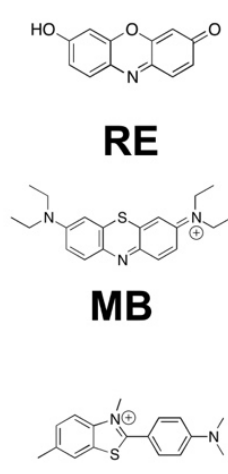

ThT

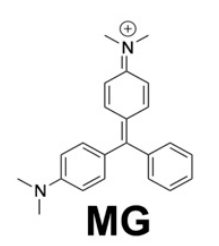

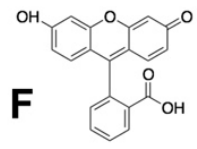
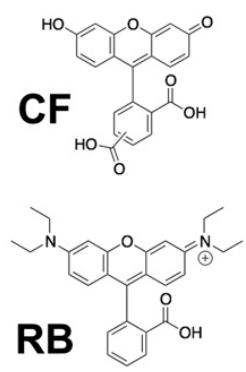

TA

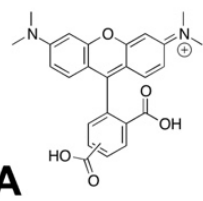

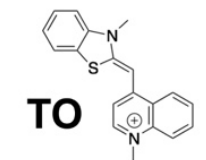

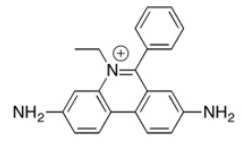

EB

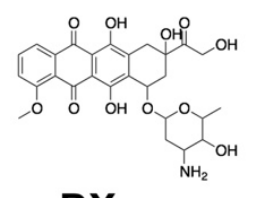

DX
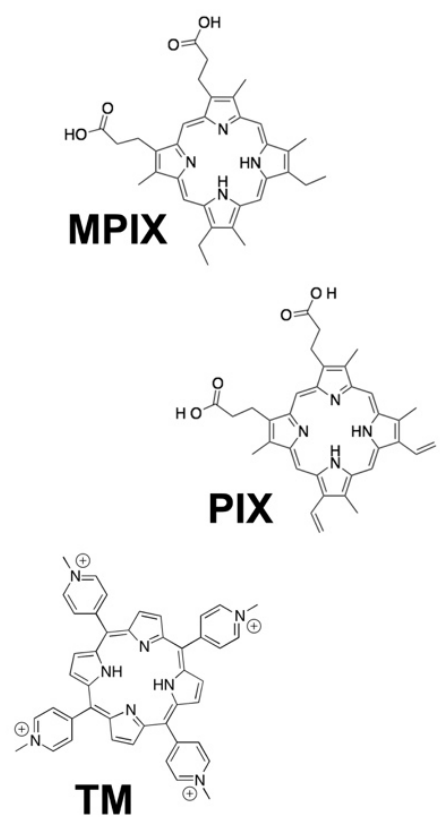

Figure 2. Fluorescent dyes used in this study: Resorufin (RE), Methylene Blue (MB), Thioflavin T (ThT), Malachite Green (MG), Fluorescein (F), 5(6)-carboxyfluorescein, mixed isomers (CF), Rhodamine B (RB), 5(6)-carboxytetramethylrhodamine, mixed isomer (TA), Thiazole Orange (TO), Ethidium Bromide (EB), Doxorubicin (DX), Mesoporphyrin IX (MPIX), Protoporphyrin IX (PIX), TmPyP4 (TM).

Previously, we reported that the main encapsulation mechanism was physical absorption (i.e., diffusion) of dyes inside SHS particles. ${ }^{7}$ Consequently, the affinity of a dye towards the SHS particles should be affected by physicochemical characteristics, such as aromatic area, ionic charge and size. We hypothesized that the main differences in affinity of these dyes towards SHS particles is determined by both the structural modifications of the G-derivatives and the resulting properties of the assembly (e.g., size, Z-potential) at the mesoscopic level. Given this, we will use molecular descriptors to correlate the characteristics of the molecules that affect the resulting affinities towards the SHS particles. 
Flow cytometry studies
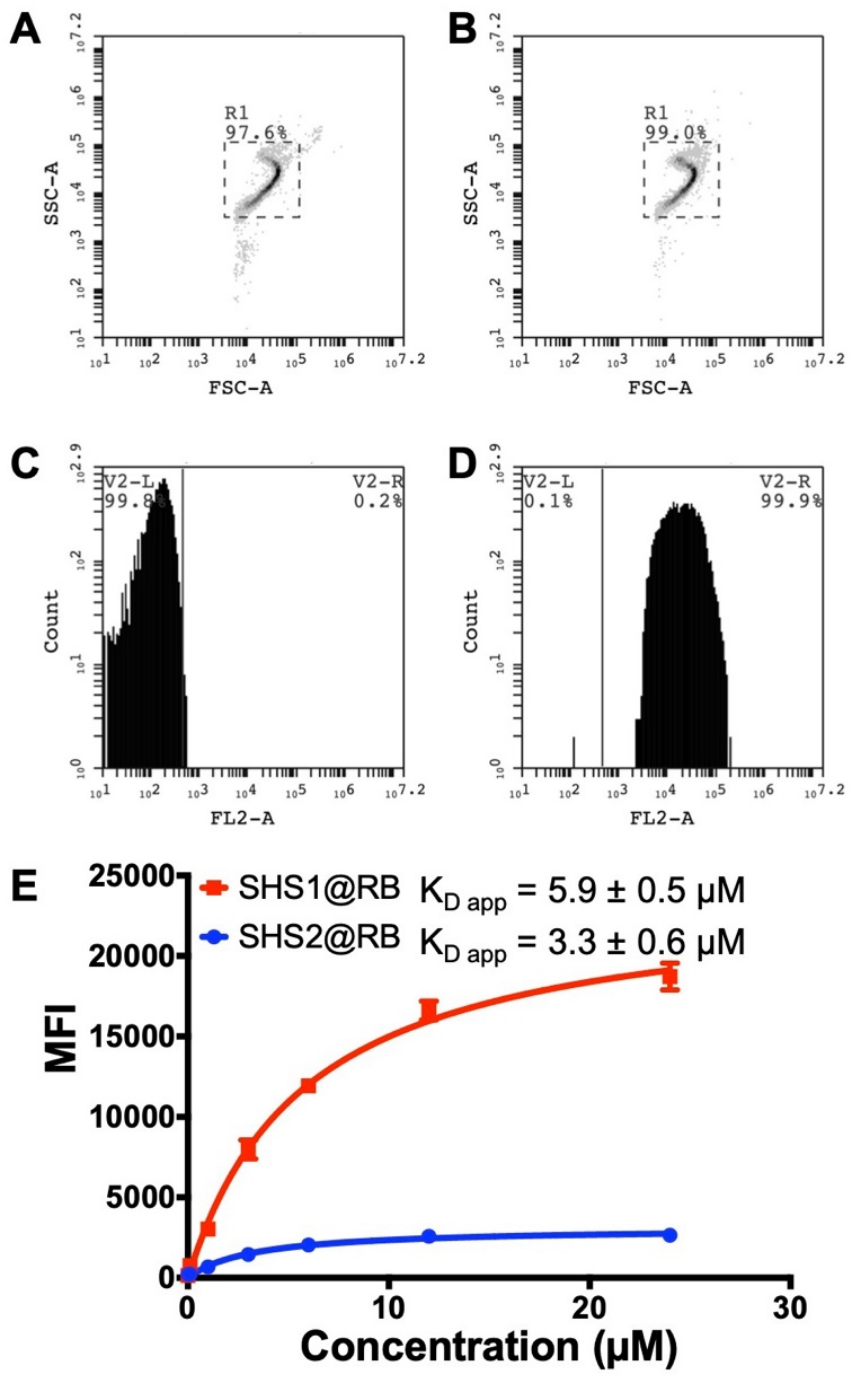

Figure 3. Flow cytometry data and saturation binding curves for the encapsulation of RB in SHS particles. (A - B) Forward scatter versus side scatter density plots of the encapsulation of RB in SHS1 and (C -D) the corresponding histograms generated from gated density plots.

Concentration of dye: (A - C) $0 \mu \mathrm{M}$, (B - D) $24 \mu \mathrm{M}$. Measurements were done in triplicate and after $1 \mathrm{~h}$ at $40{ }^{\circ} \mathrm{C}$. The histograms were generated from a gated density plot with at least $10 \mathrm{~K}$ events recorded. The vertical line of the histograms indicates the boundary of the autofluorescence of the SHS particle. Events to the right of the line represent an increase in fluorescence due to the encapsulation of RB. Excitation laser: $488 \mathrm{~nm}$; Emission channel: FL2 or $585 \mathrm{~nm}$. (E) Saturation binding curves of the encapsulation of RB in SHS1 (red) and SHS2 (blue). Median fluorescence intensity (MFI) was plotted as a function of encapsulation concentration $(0,0.1,1,3,6,12$ and $24 \mu \mathrm{M})$. $\mathrm{K}_{\mathrm{D}}$ was calculated via curve fitting with Prism Graph Pad 6 as described in the general experimental section and were subsequently used to calculate the corresponding $\mathrm{K}_{\mathrm{A}}$ values $\left(1 / \mathrm{K}_{\mathrm{D}}\right)$. Error bars represent the average of 3 measurements. SHS1 and SHS2: $299 \mu \mathrm{M}$ of G-derivative, $121 \mathrm{mM} \mathrm{KI}, \mathrm{PBS}$ pH 7.4. 
The method to determine the affinity of dyes toward SHS particles consists of mixing different concentrations of dyes with SHS particles to form a complex (SHS\#@dye, where \# is the identifier of the constituent G-derivative used to form the SHS particle) and, using FC, measure their median fluorescence intensity (MFI) as a function of dye concentration. Depending on the type of dye in the colloidal complex, we selected the 488 or $640 \mathrm{~nm}$ excitation laser in the instrument. Figure 3 shows the encapsulation of RB in SHS1 (SHS1@RB) and SHS2 (SHS2@RB), specifically, the forward scatter (FSC) versus side scatter (SSC) flow cytometry density plots as well as histograms of SHS1. FCS provides information regarding the size of the objects or events, while SSC gives insights to the changes in internal structure, also called granularity. ${ }^{30}$ Events recorded (at least 10K) shown in density plots and gated histograms are of SHS1 before and after encapsulation of RB. Histograms generated from density plots show how the fluorescent particle population changes as a function of dye concentration. A shift in the histogram before and after the encapsulation is indicative of the formation of the SHS@RB complex. The fluorescence of the SHS@RB complex increases proportionally to RB encapsulation concentration until equilibrium or saturation is achieved. MFI recorded was plotted as a function of dye concentration to make saturation plots, which are used to calculate the dissociation constant using PRISM GraphPad (see Supporting Information for details).

Density plots and histograms of the SHS@dyes complexes

The density plots of SHS@RB complexes suggest the internal structure does not change by the encapsulation of the dye, which is like the results for most other SHS complexes (Figure 3). This may be due to the favorable $\pi-\pi$ interactions of dyes with the aromatic surface of the SGQs that make up the SHS particle. However, there are density plots from other complexes that show interesting deviations from this behavior.

For example, density plots of SHS complexes made from PIX and MPIX suggest changes in internal structure, which we hypothesized were due to dye aggregation. ${ }^{31}$ Control measurements recorded after 1 min indicate aggregation of dyes MPIX and PIX without SHS particles, which in turn suggests that the density plots of SHS@MPIX or SHS@PIX show a mixture of dye aggregation and SHS complex that will affect the affinity measurements. ${ }^{30}$ In contrast, while the density plots of SHS@TO show patterns that also suggest changes to the internal structure (e.g., supramolecular arrangements) of the SHS particles (Figure S2), the possibility that this behavior was due to dye aggregation was discarded given the lack of evidence gathered from control measurements (Figure S2). We expect future studies using other techniques, like Raman spectroscopy, may help us elucidate more details about this phenomenon.

Saturation plots from FC studies 


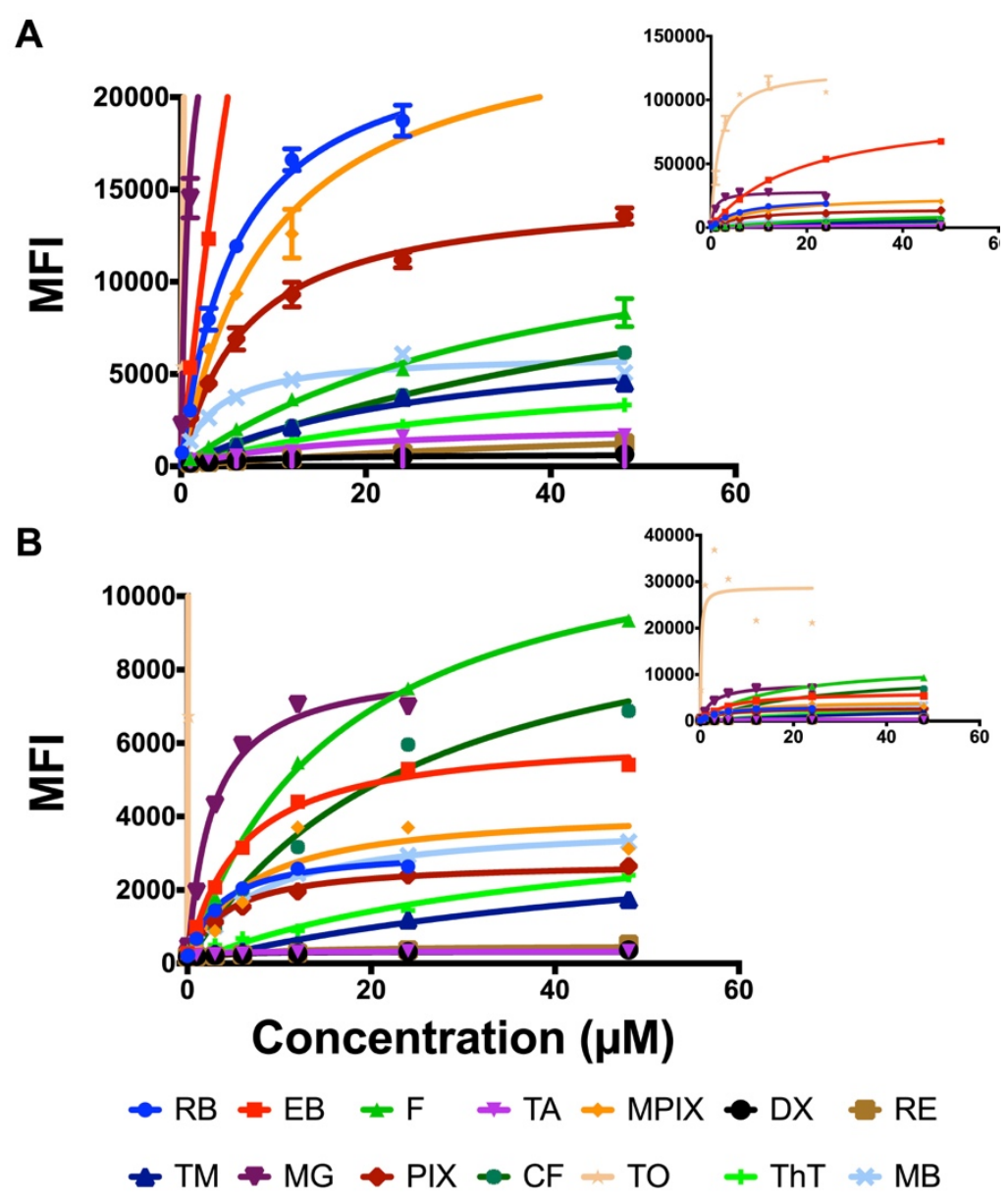

Figure 4. Saturation binding curves for the encapsulation of fluorescent dyes in (a) SHS1 and (b) SHS2. Median fluorescence intensity (MFI) was plotted as a function of concentration of the encapsulated dye. Error bars represent the average of triplicate measurements. SHS: $299 \mu \mathrm{M}$ of 1, $121 \mathrm{mM} \mathrm{KI}, \mathrm{PBS} \mathrm{pH}$ 7.4. The inset on the upper right corners depicts a zoom out of the of the saturation binding curves to the maximum MFI of (a) $150 \mathrm{~K}$ and (b) $40 \mathrm{~K}$.

The saturation binding curves in Figure 4 reveal the fluorescence intensity as a function of the concentration of the SHS@Dyes complexes after FC measurements. Previously, we saw that the encapsulation of RB in SHS1 results in higher MFI than that in SHS2. Binding curves show different MFI maxima or saturation points that are determined by the quantum yield of the dyes, the excitation maximum, or the intrinsic affinity. Here we can see that most of the complexes of SHS1 have higher MFI than SHS2, which might be due to the differences in size of these particles. These graphs were used to calculate the corresponding apparent association constants $\left(K_{\mathrm{A}}\right)$ for the SHS complexes. 
Apparent association constants

The graphs shown in Figure 5 show the results of $K_{\mathrm{A}}$ generated from the saturation graphs of SHS@Dyes complexes. If all the dyes tested had equal quantum yields, a higher MFI at a given concentration would imply a higher affinity. Since this is not the case, a simple visual assessment of the saturation does not allow a direct comparison between dyes. The shape of the curve, however, like those of dyes leading to steep initial binding curves at low concentrations result in high affinity constants (e.g., MG).

Evaluation of the overall data reveals that cationic dyes, such as TO and MG have good affinities towards SHS particles as well as zwitterionic dyes like RB and TA. Dyes TO and $\mathbf{M G}$ are known to interact with G4-DNA ${ }^{32,33}$ and other synthetic G-quadruplexes. ${ }^{34}$ In order to unveil potential relationships between dyes and their affinities towards the SHS particles we made correlation graphs of $K_{\mathrm{A}}$ as a function of various molecular descriptors for the dyes (Figures S5-S7).

Molecular descriptors, such as lipophilicity $(\log P)$, molecular weight $(\mathrm{MW})$, the expected charge of the dye at $\mathrm{pH} 7.4$, and the solvent accessible area (ASA), were plotted as a function of $K_{\mathrm{A}}$. While these plots revealed small correlations, some interesting trends surfaced. For example, for SHS1, the log $P$ shows a minor increase in affinity as a function of $K_{\mathrm{A}}$, while SHS2 shows a slight decrease instead. This may be because the SHS1 particle is more hydrophobic and thus attracts lipophilic compounds. The larger and more lipophilic nature of the vinyl-imidazole moiety attached to the meta-carbonyl group in $\mathbf{1}$ makes the corresponding assemblies more hydrophobic than those of $\mathbf{2}$, which has instead an ethoxy moiety. This is consistent with previous reports by us, ${ }^{35}$ specifically, the fact that a higher amount of salt needed to dissolve G-derivative 1 via a 'salting-in' process for self-assembly, and a decrease in the lower critical solution temperature (LCST) that promotes the formation of the SHS particles. Nevertheless, the fact that the difference in slope is small suggests that lipophilicity, as described by $\log P$, is only a minor contributor determining the native affinity towards SHS particles.

Other correlations using the descriptors of molecular weight (MW) and accessible surface area (ASA) show a slight decrease in affinity as a function of size (Figure S6). This is consistent with reports from our group showing that molecules with a large molecular mass do not diffuse completely within an SHS particle, which in turn is indicative of low affinities. ${ }^{7}$

There is a weak correlation between the affinity of the dyes with their overall charge. The Davis group reported the use of an anionic microgel made from G4-quartets that bind cationic dyes due to the structural properties of the G4 (planar conformation) and complementary charge.$^{34}$ They show that cationic dyes such as ThT, TO, MB and a derivative of $\mathbf{M G}$ (crystal violet) stabilize the gel, while RB, a non-planar zwitterionic dye 
shows weak interactions. ${ }^{34}$ In our case, both kinds of particles increase the affinity with cationic molecules. Similarly, the enhanced affinity of cationic or zwitterionic dyes towards the negatively charged SHS particles are consistent with these observations.

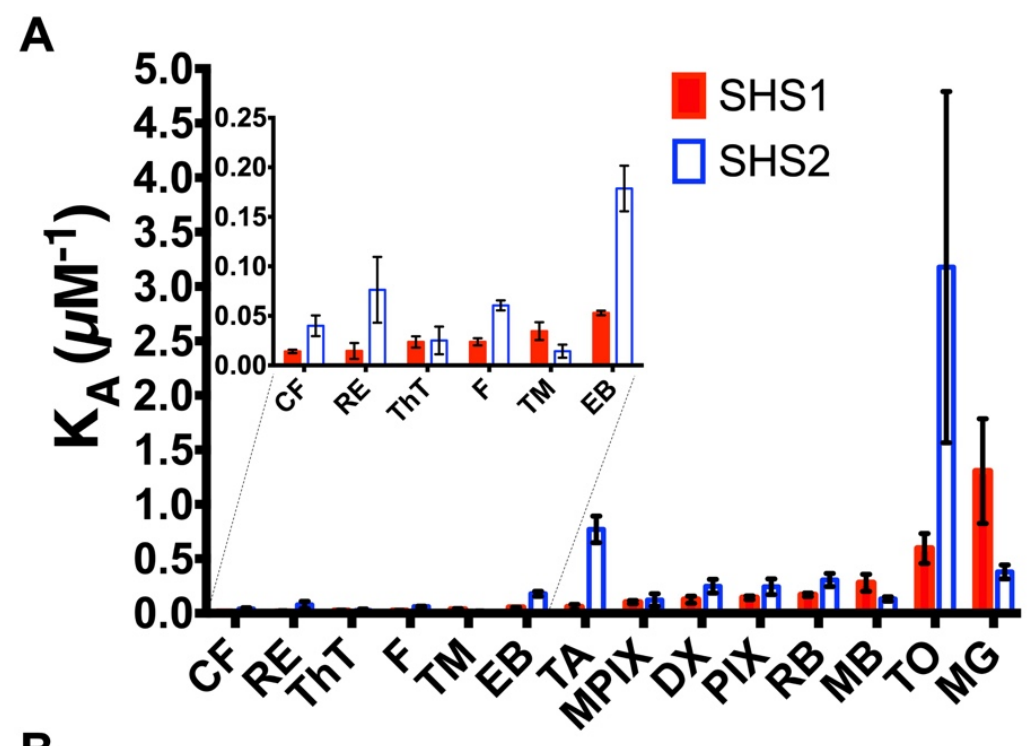

B

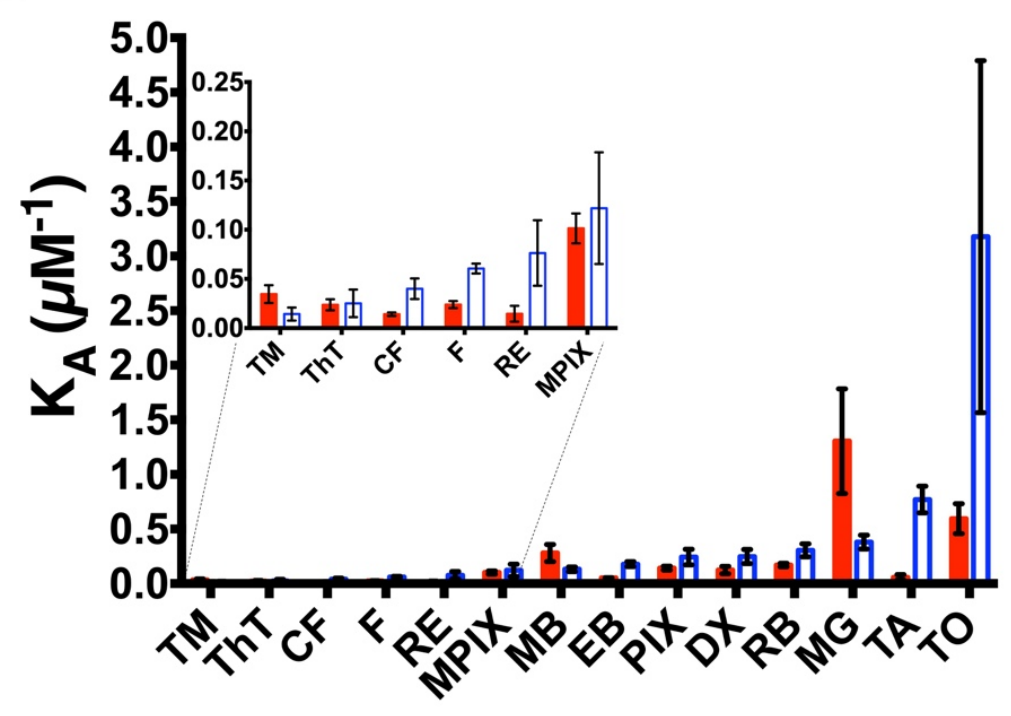

Figure 5. Apparent association constants $\left(K_{\mathrm{A}}\right)$ of fluorescent dyes towards SHS1 and SHS2 determined from Flow Cytometry measurements. $K_{\mathrm{A}}$ values are arranged in ascending order for a) SHS1 and b) SHS2. Error bars represent the standard error of the measurement reported by PRISM Graph Pad. Insets highlight the six dyes with the lowest Ka app values for each SHS.

Molecular descriptors of the dyes reveal structural affinity correlations for their affinity towards SHS particles. To further understand these trends, the affinities were analyzed based on their charge and their broad classification as derivatives of xanthene or porphyrins (Figure S8). 
The anionic xanthene dyes F and CF show low affinity towards both SHS particles tested, which is consistent with repulsive interactions due to the negative Z-potential of the particles. In contrast, the zwitterionic dyes RB and TA show moderate affinity. The highest affinities for SHS1 among the xanthene dyes were found for RB and MB, which is interesting considering that the former is neutral (zwitterionic) while the latter is cationic. In contrast, for SHS2, the dye TA shows not just the highest affinity, but also the largest difference in affinity relative to SHS1. Within the xanthene family, the overall charge of the molecules seems to be a key parameter that determines the affinity. Specifically, a negative charge seems to be an important factor determining the affinity towards these two SHS particles.

Among the three porphyrins evaluated, TM showed around an order of magnitude lower affinity than the closely related PIX and MPIX, which in turn show very similar affinities between them. This was anticipated given that what differentiates these molecules is just an unsaturated ethyl group. Relative to PIX and MPIX, porphyrin TM shows lower affinity towards SHS1, but not towards SHS2. In general, porphyrins show a propensity for binding via end-stacking to a wide variety of G4-DNA/RNA. ${ }^{27,36,37}$ In the case of TM, however, this binding mode is dampened due to the steric hindrance imposed by perpendicular arrangement of the pyridinium groups as reported in the literature, ${ }^{38}$ and corroborated by molecular modeling studies (Figure S58). We hypothesize these steric effects are also responsible for the apparent poor affinity of TM towards the constituent SGQs. Although in general cationic molecules show higher affinities towards these systems, the low affinity of TM relative to PIX and MPIX underscores the importance of other parameters. Moreover, despite PIX and MPIX having higher affinities than TM, their affinities are not significantly higher to the other anionic dyes, and generally weaker than cationic dyes. Within the set of dyes tested, there are no strong trends when evaluating the affinities of cationic dyes.

Confocal microscopy studies

CLSM provides high resolution images of cells and microparticles in two different modes: (1) differential interference contrast (DIC) and (2) fluorescence. Previously, we have used this technique to visualize the encapsulation of selected compounds in qualitative measurements. ${ }^{6,7}$ In the most basic application of CLSM, images of particles complement the FC technique to confirm the encapsulation of a target compound. However, Schuster et al. ${ }^{5}$ reported the use of an enrichment index (EI) determined by CLSM in order to quantify the efficiency of encapsulation inside liquid particles. The EI provides an alternative measurement of the partition of dyes inside and outside a colloidal particle. With this precedent in mind, we performed CSLM measurements to 
complement the affinity studies by flow cytometer and gather additional quantitative data.

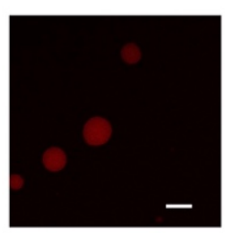

SHS1@RE

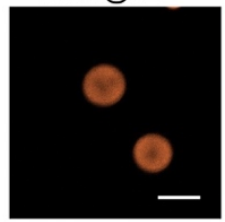

SHS1@MB

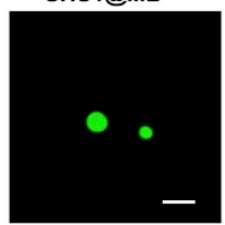

SHS1@ThT

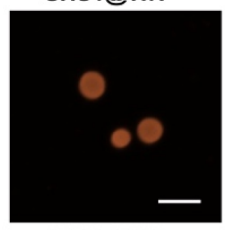

SHS1@MG

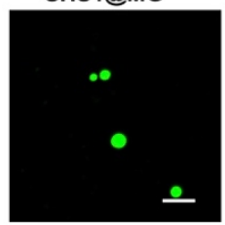

SHS1@TO

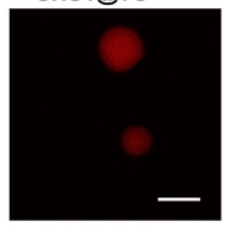

SHS1@DX

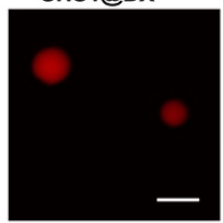

SHS1@EB

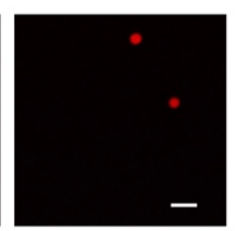

SHS2@RE

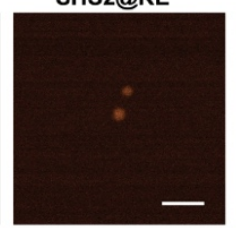

SHS2@MB

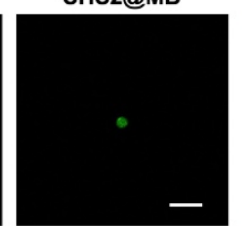

SHS2@ThT

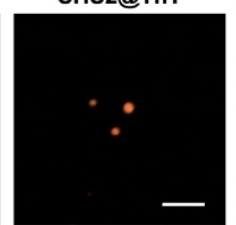

SHS2@MG

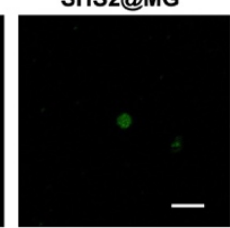

SHS2@To

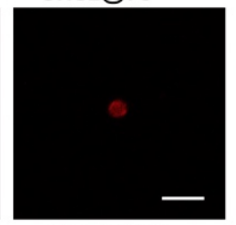

SHS2@DX

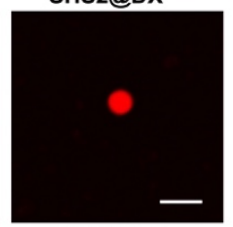

SHS2@EB

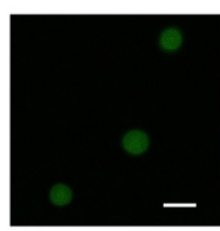

SHS1@F

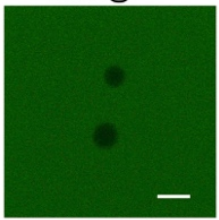

SHS1@CF

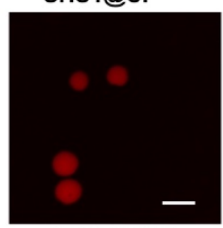

SHS1@RB

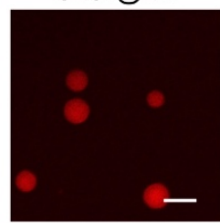

SHS1@TA

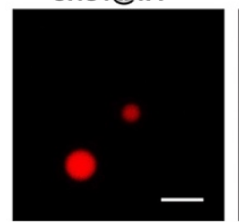

SHS1@MPIX

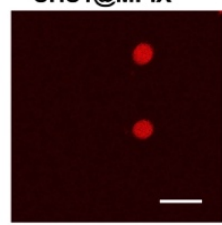

SHS1@PIX

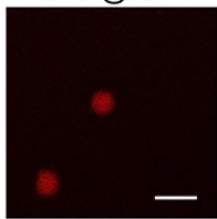

SHS1@TM

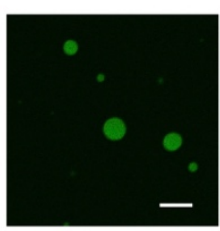

SHS2@F

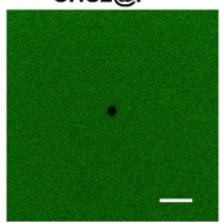

SHS2@CF

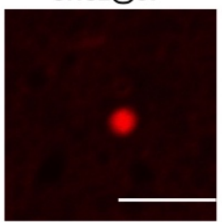

SHS2@RB

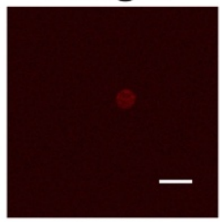

SHS2@TA

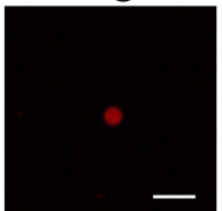

SHS2@MPIX

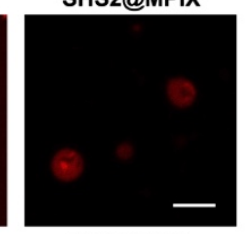

SHS2@PIX

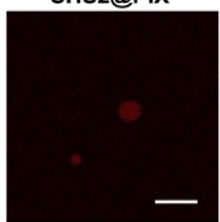

SHS2@TM

Figure 6. Confocal microscopy images for the SHS1 and SHS2 particles incubated with fluorescent dyes. All dyes were used at a concentration of $3 \mu \mathrm{M}$ except for CF $(12 \mu \mathrm{M})$. The laser used for F, CF, MPIX, PIX, TM, EB, ThT, TO, DX was $488 \mathrm{~nm}$, for RB, TA, RE it was 561 $\mathrm{nm}$, and for MG and MB it was $640 \mathrm{~nm}$. SHS1 and SHS2: $299 \mu \mathrm{M}$ of G-derivative, $121 \mathrm{mM} \mathrm{KI}$, PBS pH 7.4. 

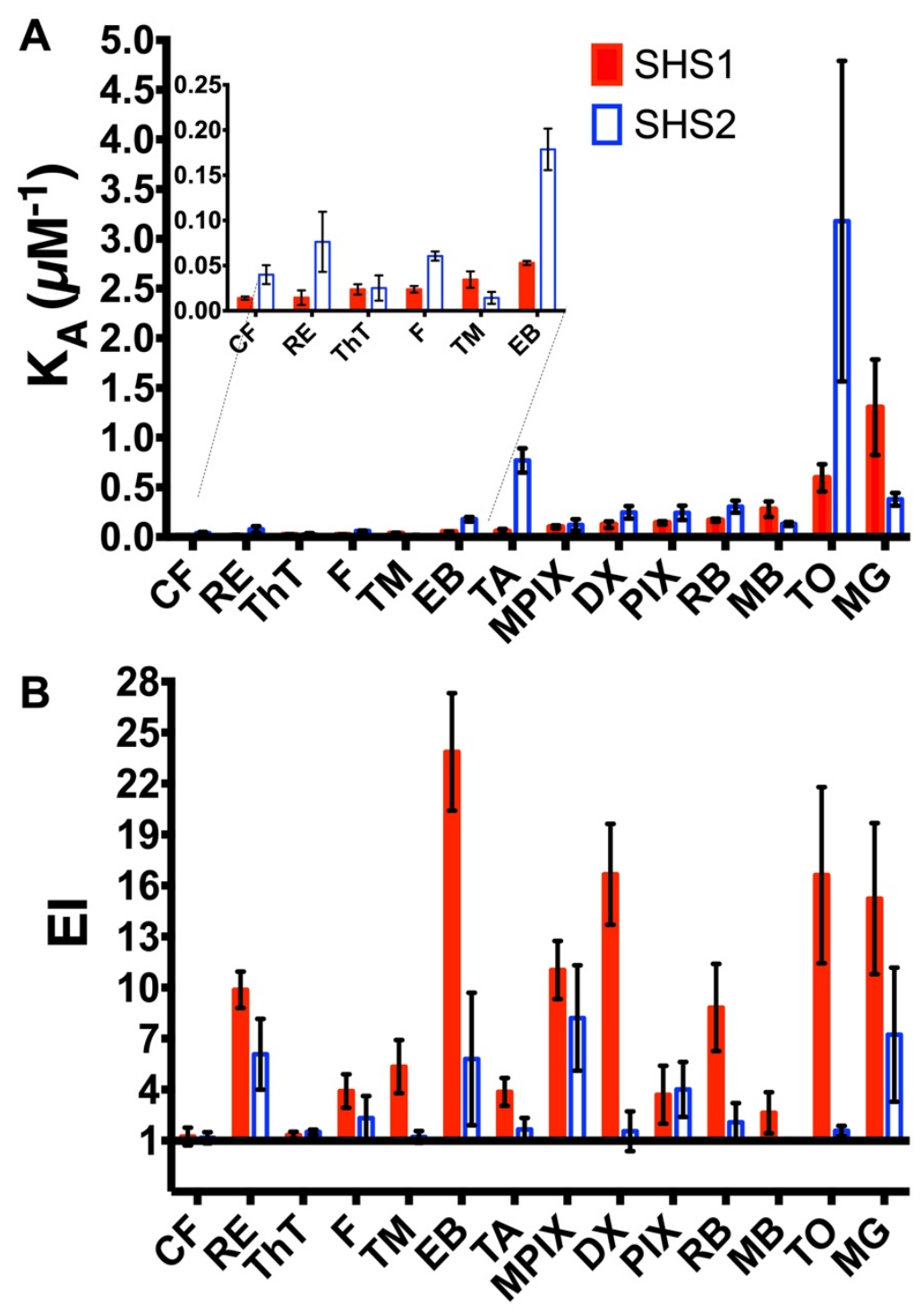

Figure 7. Flow cytometry and confocal microscopy reveal distinct quantitative information about the binding of fluorescent dyes towards SHS particles. (a) Apparent association constants $\left(K_{\mathrm{A}}\right)$ of fluorescent dyes towards SHS1 and SHS2 determined from Flow Cytometry measurements. Error bars represent the standard error of the measurement reported by PRISM Graph Pad. (b) The Enrichment Index (EI) of dyes in SHS1 and SHS2 determined by confocal microscopy data. El was calculated as the ratio of the fluorescence inside and outside of the SHS particle. The dye concentration used for the El experiments was $3 \mu \mathrm{M}$. Error bars represent the average of at least three SHS particles. SHS1 and SHS2 concentrations are $299 \mu \mathrm{M}$ in $121 \mathrm{KI}, \mathrm{PBS}$ pH 7.4. All samples were incubated for $1 \mathrm{~h}$ with or without dye. RB, TA, RE were excited using a 561 nm laser. F, DX, EB, TM, PIX, MPIX, ThT, TO were excited using $488 \mathrm{~nm}$ laser. MG, MB were excited with the $640 \mathrm{~nm}$ laser. For further details see the supporting information. 
The images obtained from CLSM measurements are consistent with the FC results showing that, except for CF, all other dyes partition preferentially within (i.e., are enriched) the SHS particles (Figure 6). Even at higher concentrations (12 $\mu \mathrm{M}), \mathbf{C F}$ is excluded from the SHS particles, which agrees well with the apparent low affinity determined with the FC method. The resulting El for the SHS@Dyes complexes showed that in most cases, for most of the dyes tested, the enrichment is higher in the larger SHS1 particles relative to the smaller SHS2 (Figure 7), which likely reflect the smaller volume for the latter.

The CLSM images also reveal differences in the distribution of dyes within the particles. Specifically, cationic dyes MB and MG appear to concentrate on the periphery of the SHS1 particles, which we hypothesize might be due to an interfacial aggregation/crystallization process. Further studies using a larger set of dyes and alternative spectroscopic techniques will be required to elucidate more details about this phenomenon.

Both FC and CLSM are single particle detection techniques that complement each other, but in general, the former has multiple advantages over the latter. The FC technique can differentiate between encapsulated dye and no dye, while CLSM was not sensitive enough to make these distinctions with some dyes (e.g., PIX) as demonstrated by control measurements shown in Figure S9. Also, the affinity constants calculated from flow cytometry appear to be unaffected by the differences in size of the particles studied. In contrast, the enrichment index shows a direct correlation between high enrichment and size. This disadvantage is compounded by the fact that the $\mathrm{El}$ is calculated from a relatively small number of measurements. While increasing the number of particles considered in the calculations of $\mathrm{El}$ is possible, time and other technical constraints (e.g., photobleaching) makes it impractical to get more than a small percentage of the thousands of events measured via FC. The FC method also allows us to compare between dyes in a high-throughput manner while confocal microscopy has certain parameters and adjustments per dye that make the measurements more time consuming. ${ }^{39}$

\section{Conclusions}

We described a method to determine the affinity constants of dyes towards SHS particles using FC and analyzed the results with the help of molecular descriptors. We observed that small modifications in the G-derivatives slightly affect the affinity of the dyes depending on its hydrophobicity or the lipophilicity of the fluorescent molecules. In 
general, cationic, small molecules, like MG and TO, show good affinity towards the SHS particles likely due to their electrostatic attraction negative Z-potential. Nevertheless, changing the size of a SHS particle can affect the loading capacity for a specific dye, but not necessarily its affinity. We also showed that confocal microscopy studies confirm the selective partition of most dyes from the bulk solution to within the SHS particles.

We are currently applying this method for the evaluation of the affinity of proteins towards SHS particles. We are also evaluating the formation SHS particles made with G-derivatives modified with affinity ligands to enhance and modulate the corresponding native affinities. Results for such studies will be reported soon.

\section{Experimental}

For experimental details see supporting information.

\section{References}

(1) Kraft, J. C.; Freeling, J. P.; Wang, Ziyao.; Ho, R. J. Y. Emerging Research and Clinical Development Trends of Liposome and Lipid Nanoparticle Drug Delivery Systems. J. Pharm. Sci. 2014, 103 (1), 29-52. https://doi.org/10.1002/jps.23773.

(2) Heyes, J.; Palmer, L.; Bremner, K.; MacLachlan, I. Cationic Lipid Saturation Influences Intracellular Delivery of Encapsulated Nucleic Acids. J. Controlled Release 2005, 107 (2), 276-287. https://doi.org/10.1016/j.jconrel.2005.06.014.

(3) Burke, P. A.; Pun, S. H.; Reineke, T. M. Advancing Polymeric Delivery Systems Amidst a Nucleic Acid Therapy Renaissance. ACS Macro Lett. 2013, 2 (10), 928934. https://doi.org/10.1021/mz400418j.

(4) Canton, I.; Battaglia, G. Endocytosis at the Nanoscale. Chem. Soc. Rev. 2012, 41 (7), 2718-2739. https://doi.org/10.1039/c2cs15309b.

(5) Schuster, B. S.; Reed, E. H.; Parthasarathy, R.; Jahnke, C. N.; Caldwell, R. M.; Bermudez, J. G.; Ramage, H.; Good, M. C.; Hammer, D. A. Controllable Protein Phase Separation and Modular Recruitment to Form Responsive Membraneless Organelles. Nat. Commun. 2018, 9 (1), 2985. https://doi.org/10.1038/s41467-01805403-1. 
(6) Betancourt, J. E.; Subramani, C.; Serrano-Velez, J. L.; Rosa-Molinar, E.; Rotello, V. M.; Rivera, J. M. Drug Encapsulation within Self-Assembled Microglobules Formed by Thermoresponsive Supramolecules. Chem. Commun. 2010, 46 (45), 8537. https://doi.org/10.1039/c0cc04063k.

(7) Negrón, L. M.; Díaz, T. L.; Ortiz-Quiles, E. O.; Dieppa-Matos, D.; Madera-Soto, B.; Rivera, J. M. Organic Nanoflowers from a Wide Variety of Molecules Templated by a Hierarchical Supramolecular Scaffold. Langmuir 2016, 32 (10), 2283-2290. https://doi.org/10.1021/acs.langmuir.5b03946.

(8) Santos, S.; Ramírez, M.; Miranda, E.; Reyes, N.; Martínez, O.; Acosta-Santiago, M.; Rivera, J. M.; Otero, M. Enhancement of Immune Responses by GuanosineBased Particles in DNA Plasmid Formulations against Infectious Diseases. J. Immunol. Res. 2019, 2019, 3409371. https://doi.org/10.1155/2019/3409371.

(9) Mason, A. F.; Buddingh', B. C.; Williams, D. S.; van Hest, J. C. M. Hierarchical Self-Assembly of a Copolymer-Stabilized Coacervate Protocell. J. Am. Chem. Soc. 2017, 139 (48), 17309-17312. https://doi.org/10.1021/jacs.7b10846.

(10) Wu, H.; Zhu, L.; Torchilin, V. P. PH-Sensitive Poly(Histidine)-PEG/DSPE-PEG CoPolymer Micelles for Cytosolic Drug Delivery. Biomaterials 2013, 34 (4), 12131222. https://doi.org/10.1016/j.biomaterials.2012.08.072.

(11) Broaders, K. E.; Cohen, J. A.; Beaudette, T. T.; Bachelder, E. M.; Frechet, J. M. J. Acetalated Dextran Is a Chemically and Biologically Tunable Material for Particulate Immunotherapy. Proc. Natl. Acad. Sci. 2009, 106 (14), 5497-5502. https://doi.org/10.1073/pnas.0901592106.

(12) Wu, D.; Wang, C.; Yang, J.; Wang, H.; Han, H.; Zhang, A.; Yang, Y.; Li, Q. Improving the Intracellular Drug Concentration in Lung Cancer Treatment through the Codelivery of Doxorubicin and MiR-519c Mediated by Porous PLGA Microparticle. Mol. Pharm. 2016, 13 (11), 3925-3933. https://doi.org/10.1021/acs.molpharmaceut.6b00702.

(13) Kim, S.-H.; Kim, J. W.; Kim, D.-H.; Han, S.-H.; Weitz, D. A. Polymersomes Containing a Hydrogel Network for High Stability and Controlled Release. Small 2013, 9 (1), 124-131. https://doi.org/10.1002/smll.201201709.

(14) Wang, L.; Chierico, L.; Little, D.; Patikarnmonthon, N.; Yang, Z.; Azzouz, M.; Madsen, J.; Armes, S. P.; Battaglia, G. Encapsulation of Biomacromolecules within Polymersomes by Electroporation. Angew. Chem. Int. Ed. 2012, 51 (44), 11122-11125. https://doi.org/10.1002/anie.201204169. 
(15) Klein, I. A.; Boija, A.; Afeyan, L. K.; Hawken, S. W.; Fan, M.; Dall'Agnese, A.; Oksuz, O.; Henninger, J. E.; Shrinivas, K.; Sabari, B. R.; Sagi, I.; Clark, V. E.; Platt, J. M.; Kar, M.; McCall, P. M.; Zamudio, A. V.; Manteiga, J. C.; Coffey, E. L.; Li, C. H.; Hannett, N. M.; Guo, Y. E.; Decker, T.-M.; Lee, T. I.; Zhang, T.; Weng, J.-K.; Taatjes, D. J.; Chakraborty, A.; Sharp, P. A.; Chang, Y. T.; Hyman, A. A.; Gray, N. S.; Young, R. A. Partitioning of Cancer Therapeutics in Nuclear Condensates. Science 2020, 368 (6497), 1386-1392. https://doi.org/10.1126/science.aaz4427.

(16) Betancourt, J. E.; Rivera, J. M. Nonpolymeric Thermosensitive Supramolecules. J. Am. Chem. Soc. 2009, 131 (46), 16666-16668.

https://doi.org/10.1021/ja9070927.

(17) Negrón, L. M.; Meléndez-Contés, Y.; Rivera, J. M. Patchy Supramolecules as Versatile Tools To Probe Hydrophobicity in Nanoglobular Systems. J. Am. Chem. Soc. 2013, 135 (10), 3815-3817. https://doi.org/10.1021/ja401373h.

(18) Hema Sagar, G.; Tiwari, M. D.; Bellare, J. R. Flow Cytometry As a Novel Tool to Evaluate and Separate Vesicles Using Characteristic Scatter Signatures. J. Phys. Chem. B 2010, 114 (31), 10010-10016. https://doi.org/10.1021/jp1027433.

(19) Keller, M.; Mahuroof, S. A.; Hong Yee, V.; Carpenter, J.; Schindler, L.; Littmann, T.; Pegoli, A.; Hübner, H.; Bernhardt, G.; Gmeiner, P.; Holliday, N. D.

Fluorescence Labeling of Neurotensin(8-13) via Arginine Residues Gives Molecular Tools with High Receptor Affinity. ACS Med. Chem. Lett. 2020, 11 (1), 16-22. https://doi.org/10.1021/acsmedchemlett.9b00462.

(20) Stein, R. A.; Wilkinson, J. C.; Guyer, C. A.; Staros, J. V. An Analytical Approach to the Measurement of Equilibrium Binding Constants: Application to EGF Binding to EGF Receptors in Intact Cells Measured by Flow Cytometry ${ }^{\dagger}$. Biochemistry 2001, 40 (20), 6142-6154. https://doi.org/10.1021/bi002817a.

(21) Semmling, M.; Kreft, O.; Muñoz Javier, A.; Sukhorukov, G. B.; Käs, J.; Parak, W. J. A Novel Flow-Cytometry-Based Assay for Cellular Uptake Studies of Polyelectrolyte Microcapsules. Small 2008, 4 (10), 1763-1768. https://doi.org/10.1002/smll.200800596.

(22) Vardanyan, I.; Navoyan, Z.; Moya, S. E.; Arakelyan, V.; Donath, E. Flow Cytometry Study of the Non-Fickean Diffusion of Small-Charged Molecules in Poly(Diallyl Dimethyl Ammonium Chloride)/Poly(Styrene Sodium Sulfonate) 
Multilayers: Impact of the Layer Number, Top Layer, and Concentration of Diffusing Molecules. J. Phys. Chem. B 2019, 123 (9), 2182-2189.

https://doi.org/10.1021/acs.jpcb.8b12367.

(23) Temmerman, K.; Nickel, W. A Novel Flow Cytometric Assay to Quantify Interactions between Proteins and Membrane Lipids. J. Lipid Res. 2009, 50 (6), 1245-1254. https://doi.org/10.1194/jlr.D800043-JLR200.

(24) Liu, X.; Bing, T.; Shangguan, D. Microbead-Based Platform for Multiplex Detection of DNA and Protein. ACS Appl. Mater. Interfaces 2017, 9 (11), 9462-9469. https://doi.org/10.1021/acsami.7b00418.

(25) Rivera, J. M.; Negrón, L. M. Supramolecular Hacky Sacks (SHS), Method of Synthesis and Applications Thereof. 10106572, 2018.

(26) Honary, S.; Zahir, F. Effect of Zeta Potential on the Properties of Nano-Drug Delivery Systems - A Review (Part 1). Trop. J. Pharm. Res. 2013, 12 (2), 255264. https://doi.org/10.4314/tjpr.v12i2.19.

(27) Han, H.; Langley, D. R.; Rangan, A.; Hurley, L. H. Selective Interactions of Cationic Porphyrins with G-Quadruplex Structures. J. Am. Chem. Soc. 2001, 123 (37), 8902-8913. https://doi.org/10.1021/ja002179j.

(28) Murat, P.; Singh, Y.; Defrancq, E. Methods for Investigating G-Quadruplex DNA/Ligand Interactions. Chem. Soc. Rev. 2011, 40 (11), 5293. https://doi.org/10.1039/c1cs15117g.

(29) Vummidi, B. R.; Alzeer, J.; Luedtke, N. W. Fluorescent Probes for G-Quadruplex Structures. ChemBioChem 2013, 14 (5), 540-558. https://doi.org/10.1002/cbic.201200612.

(30) Adan, A.; Alizada, G.; Kiraz, Y.; Baran, Y.; Nalbant, A. Flow Cytometry: Basic Principles and Applications. Crit. Rev. Biotechnol. 2017, 37 (2), 163-176. https://doi.org/10.3109/07388551.2015.1128876.

(31) Scolaro, L. M.; Castriciano, M.; Romeo, A.; Patanè, S.; Cefalì, E.; Allegrini, M. Aggregation Behavior of Protoporphyrin IX in Aqueous Solutions: Clear Evidence of Vesicle Formation. J. Phys. Chem. B 2002, 106 (10), 2453-2459. https://doi.org/10.1021/jp013155h. 
(32) Monchaud, D.; Allain, C.; Teulade-Fichou, M.-P. Thiazole Orange: A Useful Probe for Fluorescence Sensing of G-Quadruplex-Ligand Interactions. Nucleosides Nucleotides Nucleic Acids 2007, 26 (10-12), 1585-1588. https://doi.org/10.1080/15257770701548212.

(33) Bhasikuttan, A. C.; Mohanty, J.; Pal, H. Interaction of Malachite Green with Guanine-Rich Single-Stranded DNA: Preferential Binding to a G-Quadruplex. Angew. Chem. Int. Ed. 2007, 46 (48), 9305-9307. https://doi.org/10.1002/anie.200703251.

(34) Peters, G. M.; Skala, L. P.; Davis, J. T. A Molecular Chaperone for G4-Quartet Hydrogels. J. Am. Chem. Soc. 2016, 138 (1), 134-139. https://doi.org/10.1021/jacs.5b08769.

(35) Acosta-Santiago, M. Biologically Relevant Formulation and Expansion of Responsive Properties of Guanine Based Supramolecular Particles. Ph.D., Universidad de Puerto Rico, San Juan, P.R., 2021.

(36) Wei, C.; Jia, G.; Yuan, J.; Feng, Z.; Li, C. A Spectroscopic Study on the Interactions of Porphyrin with G-Quadruplex DNAs ${ }^{\dagger}$. Biochemistry 2006, 45 (21), 6681-6691. https://doi.org/10.1021/bi052356z.

(37) Wheelhouse, R. T.; Sun, D.; Han, H.; Han, F. X.; Hurley, L. H. Cationic Porphyrins as Telomerase Inhibitors: The Interaction of Tetra-( $N$-Methyl-4-Pyridyl)Porphine with Quadruplex DNA. J. Am. Chem. Soc. 1998, 120 (13), 3261-3262. https://doi.org/10.1021/ja973792e.

(38) Parkinson, G. N.; Ghosh, R.; Neidle, S. Structural Basis for Binding of Porphyrin to Human Telomeres. Biochemistry 2007, 46 (9), 2390-2397. https://doi.org/10.1021/bi062244n.

(39) Danaei, M.; Kalantari, M.; Raji, M.; Samareh Fekri, H.; Saber, R.; Asnani, G. P.; Mortazavi, S. M.; Mozafari, M. R.; Rasti, B.; Taheriazam, A. Probing Nanoliposomes Using Single Particle Analytical Techniques: Effect of Excipients, Solvents, Phase Transition and Zeta Potential. Heliyon 2018, 4 (12), e01088. https://doi.org/10.1016/j.heliyon.2018.e01088. 